\title{
The impact of relative hypotension on acute kidney injury progression after cardiac surgery: a multicenter retrospective cohort study
}

Yuki Kotani ${ }^{1}$, Takuo Yoshida ${ }^{2 *}$, Junji Kumasawa ${ }^{3}$, Jun Kamei ${ }^{4}$, Akihisa Taguchi ${ }^{5}$, Koji Kido ${ }^{6}$, Naoki Yamaguchi ${ }^{7}$, Takayuki Kariya ${ }^{8}$, Masato Nakasone ${ }^{9}$, Noriko Mikami ${ }^{10}$, Takahiro Koga ${ }^{11}$, Izumi Nakayama ${ }^{12}$, Mami Shibata ${ }^{13}$, Tomonao Yoshida ${ }^{14}$, Hiroshi Nashiki ${ }^{15}$, Shinsuke Karatsu ${ }^{16}$, Kazutaka Nogi ${ }^{17}$, Natsuko Tokuhira ${ }^{18}$ and Junichi Izawa ${ }^{19}$

\begin{abstract}
Background: Cardiac surgery is performed worldwide, and acute kidney injury (AKI) following cardiac surgery is a risk factor for mortality. However, the optimal blood pressure target to prevent AKI after cardiac surgery remains unclear. We aimed to investigate whether relative hypotension and other hemodynamic parameters after cardiac surgery are associated with subsequent AKI progression.

Methods: We retrospectively enrolled adult patients admitted to 14 intensive care units after elective cardiac surgery between January and December 2018. We defined mean perfusion pressure (MPP) as the difference between mean arterial pressure (MAP) and central venous pressure (CVP). The main exposure variables were time-weighted-average MPP-deficit (i.e., the percentage difference between preoperative and postoperative MPP) and time spent with MPPdeficit $>20 \%$ within the first $24 \mathrm{~h}$. We defined other pressure-related hemodynamic parameters during the initial $24 \mathrm{~h}$ as exploratory exposure variables. The primary outcome was AKI progression, defined as one or more AKI stages using Kidney Disease: Improving Global Outcomes' creatinine and urine output criteria between 24 and 72 h. We used multivariable logistic regression analyses to assess the association between the exposure variables and AKI progression.

Results: Among the 746 patients enrolled, the median time-weighted-average MPP-deficit was 20\% [interquartile range (IQR): 10-27\%], and the median duration with MPP-deficit >20\% was $12 \mathrm{~h}$ (IQR: 3-20 h). One-hundred-andtwenty patients (16.1\%) experienced AKI progression. In the multivariable analyses, time-weighted-average MPPdeficit or time spent with MPP-deficit > 20\% was not associated with AKI progression [odds ratio (OR): 1.01, 95\% confidence interval (95\% Cl): 0.99-1.03]. Likewise, time spent with MPP-deficit $>20 \%$ was not associated with AKI progression (OR: 1.01, 95\% Cl 0.99-1.04). Among exploratory exposure variables, time-weighted-average CVP, timeweighted-average MPP, and time spent with MPP $<60 \mathrm{mmHg}$ were associated with AKI progression $(\mathrm{OR}: 1.12,95 \% \mathrm{Cl}$ 1.05-1.20; OR: 0.97, 95\% Cl 0.94-0.99; OR: 1.03, 95\% Cl 1.00-1.06, respectively).
\end{abstract}

Conclusions: Although higher CVP and lower MPP were associated with AKI progression, relative hypotension was not associated with AKI progression in patients after cardiac surgery. However, these findings were based on exploratory investigation, and further studies for validating them are required.

\footnotetext{
*Correspondence: hawaii28981@yahoo.co.jp

2 Department of Intensive Care Medicine, Tokyo Women's Medical

University, 8-1 Kawada-cho, Shinjuku-ku, Tokyo 162-8666, Japan

Full list of author information is available at the end of the article
}

\section{Springer Open}

(c) The Author(s) 2021. Open Access This article is licensed under a Creative Commons Attribution 4.0 International License, which permits use, sharing, adaptation, distribution and reproduction in any medium or format, as long as you give appropriate credit to the original author(s) and the source, provide a link to the Creative Commons licence, and indicate if changes were made. The images or other third party material in this article are included in the article's Creative Commons licence, unless indicated otherwise in a credit line to the material. If material is not included in the article's Creative Commons licence and your intended use is not permitted by statutory regulation or exceeds the permitted use, you will need to obtain permission directly from the copyright holder. To view a copy of this licence, visit http://creativecommons.org/licenses/by/4.0/. 
Trial Registration UMIN-CTR, https://www.umin.ac.jp/ctr/index-j.htm, UMIN000037074.

Keywords: Acute kidney injury, Blood pressure, Cardiac surgery, Cardiogenic shock, Critical care, Hemodynamics

\section{Background}

Cardiac surgery is performed worldwide [1-5]. Acute kidney injury (AKI) is a major complication of cardiac surgery. Cardiac surgery-associated AKI (CSA-AKI) occurs in approximately one-third of patients after cardiac surgery and is associated with mortality and morbidity [6-9]. However, there is no effective strategy supported by robust evidence to prevent or treat CSAAKI [10].

Although blood pressure (BP) is a vital determinant of renal perfusion, the optimal BP target to prevent AKI progression remains unclear for intensive care unit (ICU) patients. Previous randomized controlled trials (RCTs) assessed different absolute mean arterial pressure (MAP) targets [11, 12]. Subgroup analysis of one trial showed that chronic hypertensive patients in the high MAP target group had a lower incidence of AKI [11]. However, these trials showed no difference in mortality between different MAP targets in septic or vasodilatory shock for the overall patient population $[11,12]$. Mean perfusion pressure (MPP), which is the difference between MAP and central venous pressure (CVP), can also be used as a surrogate marker for renal perfusion because elevated CVP has been reported as a risk factor for AKI in ICU patients [13, 14]. Although CVP monitoring is readily available in patients after cardiac surgery [15], few studies have assessed the relationship between MPP and AKI in this population [16, 17].

Moreover, relative hypotension, defined as a deficit between the premorbid and achieved BP, has been investigated as a BP target to prevent AKI progression. The Kidney Disease: Improving Global Outcome (KDIGO) guidelines suggest that the optimal BP target may vary according to the premorbid level [18]. Several studies have shown the association of relative hypotension with AKI progression in patients with sepsis or shock [19-21]. Although chronic hypertension is common among cardiac surgery patients [22-24], the only available evidence is a single-center study showing that patients with AKI progression had greater relative hypotension [25].

Accordingly, we conducted a multicenter retrospective cohort study to test whether the relative hypotension using MPP during the first $24 \mathrm{~h}$ would be associated with AKI progression between 24 and $72 \mathrm{~h}$ among postoperative cardiac surgery patients. We also explored the relationship between other BP-related parameters and subsequent AKI progression.

\section{Methods}

The Blood pressure and Relative Optimal Target after Heart surgery in Epidemiologic Registry (BROTHER) study was a multicenter retrospective cohort study enrolling adult patients after cardiac surgery in 14 ICUs in Japan (UMIN-CTR; trial ID: UMIN000037074). All study patients were followed up until hospital discharge or 30 days after the surgery, whichever came later. The ethics committee of Kameda Medical Center (No. 19-013) and the ethics committees of all other participating hospitals approved this study with an opt-out policy from the patient or proxy. The need for informed consent was waived due to the retrospective nature of this study.

\section{Participants and data sources/measurement}

We consecutively included all adult patients aged 18 years or older who were admitted to one of the ICUs after elective coronary artery bypass grafting (CABG) or valve surgery between January 1st and December 31st in 2018. We excluded (i) patients who were discharged from the ICU within $24 \mathrm{~h}$; (ii) patients who underwent emergency cardiac surgery; (iii) patients on extracorporeal membrane oxygenation, intra-aortic balloon pumping, or ventricular assist device during the first $24 \mathrm{~h}$ after ICU admission; (iv) patients with postoperative BP recorded at an interval of longer than $1 \mathrm{~h}$; (v) patients without available BP recordings within 365 days before the surgery; (vi) patients who opted out of this study; and (vii) patients censored for data collection. We defined emergency cardiac surgery as urgent surgery for a life-threatening condition to save life or prevent organ dysfunction. We excluded patients after emergency cardiac surgery because we assumed that detailed information about preoperative blood pressure was often not available, and the calculation of MPP deficiency would be inaccurate.

We collected demographic data, comorbidities, Acute Physiology and Chronic Health Evaluation (APACHE) II score [26], Sequential Organ Failure Assessment score [27], operative characteristics (e.g., type of surgery, surgery time, cardiopulmonary bypass time, transfusion, hemorrhage, and fluid balance), and vasopressor, inotrope, and diuretic use within $24 \mathrm{~h}$ after ICU admission. We describe the definitions of variables and details of data collection in Additional file 1: Table S1 and Fig. S1, respectively.

We defined MPP as the difference between MAP and CVP. The preoperative BP was defined as the average of three recent $\mathrm{BP}$ readings recorded at least one day apart 
within 1 year before the surgery. We calculated preoperative MAP as diastolic $\mathrm{BP}+($ systolic $\mathrm{BP}$ - diastolic $\mathrm{BP}) / 3$. The preoperative CVP was estimated using inferior vena cava data from preoperative echocardiographic findings according to the 2010 American Society of Echocardiography guidelines [28]. When preoperative echocardiography was unavailable, we assumed the mean CVP values stratified for presence or absence of heart disease [29], as previously reported $[21,25]$. Additional file 1: Table S2 describes the detailed protocol to determine preoperative CVP.

We collected postoperative MAP and CVP at 1-h intervals during the first $24 \mathrm{~h}$ after ICU admission and assumed the CVP value as the average value every $4 \mathrm{~h}$. When there was no available CVP measurement within any 4-h interval, we imputed the CVP value closest to the timepoint.

\section{Exposure and outcome variables}

The primary exposure variable was time-weighted-average MPP-deficit. We defined MPP-deficit as (preoperative MPP-postoperative MPP)/preoperative MPP. We calculated the time-weighted-average MPP-deficit as an aggregate area-under-the-curve divided by $24 \mathrm{~h}$, where the area-under-the-curve was measured as an integrated expression over time using a positive incremental method. When the postoperative MPP was higher than the preoperative MPP, we regarded the MPP-deficit at the timepoint as zero. The secondary exposure variable was time spent with MPP-deficit $>20 \%$, a threshold considered as a significant relative reduction in BP $[19,21]$. We also included the following exploratory exposure variables: postoperative time-weighted-average MAP, CVP, MPP, time spent with MAP $<65 \mathrm{mmHg}$, time spent with $\mathrm{MPP}<60 \mathrm{mmHg}$, time-weighted-average MAP-deficit, and time spent with MAP-deficit $>20 \%$.

The primary outcome variable was AKI progression. We defined AKI progression as an increase of at least one AKI stage between 24 and $72 \mathrm{~h}$ compared to the first $24 \mathrm{~h}$. We defined patients with AKI progression as the AKI group and patients without AKI progression as the Non-AKI group. We used both the creatinine and urine output criteria of KDIGO [18]. The intensivist in charge of data collection in each participating hospital clinically determined the preoperative baseline serum creatinine level based on the medical records. The secondary outcome variables included major adverse kidney event (MAKE) within 30 days after the surgery, hospital mortality, renal replacement therapy (RRT) required during ICU stay, fluid balance on the second and third days of ICU admission, new-onset atrial fibrillation during ICU stay, stroke during the hospital stay, and mesenteric ischemia during the hospital stay. We defined MAKE30 as a composite outcome of death, new initiation of RRT, or doubling of serum creatinine from the preoperative level within 30 days after the surgery [30].

\section{Statistical methods}

Among the patients meeting the eligibility criteria, we excluded from our analysis: (i) patients with end-stage kidney disease; (ii) patients with a preoperative serum creatinine $>3.5 \mathrm{mg} / \mathrm{dL}$; (iii) patients who lacked urine output data during the first $24 \mathrm{~h}$ after ICU admission; (iv) patients with stage 3 AKI within the first $24 \mathrm{~h}$ after ICU admission; (v) patients with $<50 \%$ CVP recordings within the first $24 \mathrm{~h}$ after ICU admission, and (vi) patients who underwent reoperation during the first $24 \mathrm{~h}$ after ICU admission. In all variables except for CVP, we reported the number of missing data if we had missing data and excluded cases with missing data from each analysis. We described how to impute missing postoperative CVP values in "Participants and data source/measurement" section.

We presented categorical variables as numbers with percentages and continuous variables as medians with interquartile range. We compared baseline characteristics and hemodynamic parameters between AKI and Non-AKI groups using the Chi-square test or Fisher's exact test for categorical variables and the Mann-Whitney $U$ test for continuous variables. The impact of all exposure variables on AKI progression was assessed using multivariable logistic regression analyses adjusted for age, APACHE II score, chronic hypertension, preoperative left ventricular ejection fraction, baseline serum creatinine, surgery time, cardiopulmonary bypass (CPB), intraoperative fluid balance, and postoperative red blood cell transfusion within $24 \mathrm{~h}$ after ICU admission. We selected these variables based on the clinical relevance and their importance as risk factors for AKI in previous studies [10,31-35]. The results of multivariable logistic regression models were then plotted using marginal probabilities (average marginal effect) of outcomes across observed ranges of exposure variables to facilitate interpretation.

All analyses were conducted using Stata version 16 (StataCorp, College Station, TX, USA) and EZR (Saitama Medical Center, Jichi Medical University, ver. 1.36), which is a graphical user interface for $\mathrm{R}$ (The $\mathrm{R}$ Foundation for Statistical Computing, Vienna, Austria). We considered a two-sided $p$ value $<0.05$ as statistically significant.

We conducted three sensitivity analyses. First, the type of surgery determines the necessity of $\mathrm{CPB}$, which can influence intraoperative management [36] and is associated with postoperative AKI [33]; thus, we performed sensitivity analyses for patients with or without CPB. Second, since less relative hypotension can improve renal 
perfusion, especially in patients with chronic hypertension, given a potential rightward shift of the curve for organ pressure-flow autoregulation [37], we performed sensitivity analyses for patients with or without chronic hypertension. Third, considering that most previous studies assessing the association of relative hypotension and AKI enrolled only patients with vasopressor support $[19,21,25]$, we performed sensitivity analyses for patients with or without vasopressor support. We defined vasopressor support as receiving norepinephrine, epinephrine, dopamine, vasopressin, or phenylephrine during the first $24 \mathrm{~h}$ after ICU admission.

\section{Results}

Among 1568 adult patients admitted to the ICUs after CABG or valve surgery between January and December 2018, we registered 870 patients on the BROTHER study. During the data collection period of this study, a pandemic of COVID-19 occurred, which made data collection difficult for some investigators, and we could not enroll 42 patients. After excluding 124 patients, we analyzed 746 patients (Additional file 1: Fig. S2). No patients were lost to follow-up.

Table 1 summarizes the patient characteristics and perioperative management. One-hundred-and-twenty patients (16\%) had AKI progression. The median age was 71 years, and most patients (73\%) had chronic hypertension. Among the vasopressors and inotropes used in the first $24 \mathrm{~h}$, dobutamine was the most frequently used drug (53\%), followed by norepinephrine (40\%). The baseline serum creatinine level was higher in the AKI group. Although preoperative MAP was similar between the two groups, preoperative MPP was lower in the AKI group. Additional file 1: Table S3 describes the baseline characteristics and perioperative management in detail.

Table 2 compares the postoperative hemodynamic parameters and exposure variables between the AKI and Non-AKI groups. CVP was missing for 198 (4.4\%) out of 4,476 timepoints. While both groups achieved similar time-weighted-average MAP, the AKI group achieved a higher CVP, which resulted in a lower achieved MPP and more prolonged duration with $\mathrm{MPP}<60 \mathrm{mmHg}$ in the AKI group. Time-weighted-average MPP-deficit and time spent with MPP-deficit $>20 \%$ were comparable between the two groups (Fig. 1). Although time spent with MAP $<65 \mathrm{mmHg}$ was statistically significantly longer in the AKI group, the numerical difference was slight. Time-weighted-average MAP-deficit and time spent with MAP-deficit $>20 \%$ were similar between the two groups (Additional file 1: Fig. S3). Table 3 describes the clinical outcomes. Seven patients (0.9\%) experienced MAKE30, and hospital mortality occurred in four patients $(0.5 \%)$.
Multivariable logistic regression analyses (Additional file 1: Table S4 and Fig. 2) showed that, for every percent increase in time-weighted-average MPP-deficit, the adjusted odds ratio (OR) for AKI progression was 1.01 [95\% confidence interval (CI), 0.99-1.03; $p=0.46$ ]. Similarly, for every hour increase in time spent with MPPdeficit $>20 \%$, the adjusted OR for AKI progression was 1.01 (95\% CI $0.99-1.04 ; p=0.38$ ). Among the exploratory exposure variables, time-weighted-average CVP (adjusted OR: 1.12, 95\% CI 1.05-1.20; $p=0.0013$ ), timeweighted-average MPP (adjusted OR: 0.97, 95\% CI $0.94-$ 0.99; $p=0.0078$ ), and time spent with $\mathrm{MPP}<60 \mathrm{mmHg}$ (adjusted OR: 1.03, 95\% CI 1.00-1.06; $p=0.024$ ) were statistically significantly associated with AKI progression.

The results of the sensitivity analyses are presented in Additional file 1: Table S5. Again, we found results similar to those of the main analyses.

\section{Discussion}

Key findings

This multicenter retrospective cohort study investigated whether relative hypotension and other BP-related hemodynamic parameters were associated with subsequent AKI progression in adult patients after elective cardiac surgery. Among 746 patients eligible for this analysis, the occurrence of AKI progression was $16 \%$. We observed that relative hypotension was not associated with AKI progression, while higher CVP, lower MPP, and time spent with $\mathrm{MPP}<60 \mathrm{mmHg}$ were associated with AKI progression among exploratory exposure variables.

\section{Relationship with previous studies}

Recent studies have assessed the relationship between relative hypotension and AKI progression in critically ill patients [19-21, 25]. Among those, the only multicenter study, which enrolled 302 vasopressor-dependent patients with vasodilatory shock, showed that relative hypotension using MPP was an independent risk factor for AKI progression after adjusting for relevant confounders [21]. The only evidence for cardiac surgery patients showed that those who developed AKI progression had greater relative hypotension than those who did not [25]. However, this was a small single-center study that included only vasopressor-dependent patients and lacked multivariable analyses.

Our results were inconsistent with previous studies [21, 25]. The multivariable analyses found that relative hypotension was not associated with AKI progression, although lower postoperative MPP was significantly associated with AKI progression. The sensitivity analyses, including patients with or without vasopressor support, also showed similar results. Greater relative hypotension was caused by higher preoperative or lower postoperative 
Table 1 Patient characteristics and perioperative management

\begin{tabular}{|c|c|c|c|c|}
\hline & $\begin{array}{l}\text { All } \\
N=746\end{array}$ & $\begin{array}{l}\text { AKI group } \\
N=120\end{array}$ & $\begin{array}{l}\text { Non-AKI group } \\
N=626\end{array}$ & $p$ value \\
\hline Age, years & $71[63-77]$ & $72[64-77]$ & $70[63-77]$ & 0.58 \\
\hline Males, n (\%) & $464(62)$ & $73(61)$ & $391(63)$ & 0.76 \\
\hline Chronic hypertension, $n(\%)$ & $546(73)$ & $88(73)$ & $458(73)$ & 1.0 \\
\hline Previous cardiac surgery, n (\%) & $74(9.9)$ & $18(15)$ & $56(8.9)$ & 0.047 \\
\hline $\mathrm{LVEF}, n(\%)$ & & & & 0.72 \\
\hline$>50 \%$ & $596(80)$ & $95(79)$ & $50.1(80)$ & \\
\hline $35-50 \%$ & $120(16)$ & $22(18)$ & $98(16)$ & \\
\hline $20-34 \%$ & $28(3.8)$ & $3(2.5)$ & $25(4.0)$ & \\
\hline$<20 \%$ & $2(0.3)$ & $0(0)$ & $2(0.3)$ & \\
\hline Baseline serum creatinine, mg/dL & $0.85[0.70-1.03]$ & $0.90[0.77-1.08]$ & $0.83[0.70-1.01]$ & 0.0051 \\
\hline Baseline MPP, mmHg & $80[74-88]$ & 78 [71-85] & $81[74-88]$ & 0.020 \\
\hline Baseline MAP, mmHg & 85 [78-92] & 83 [77-89] & 85 [78-92] & 0.089 \\
\hline Baseline CVP, mmHg & $3[3-6]$ & $3[3-6]$ & $3[3-6]$ & 0.0013 \\
\hline \multicolumn{5}{|l|}{ Type of surgery, n (\%) } \\
\hline CABG & $257(35)$ & $37(30.8)$ & $220(35.1)$ & 0.40 \\
\hline Mitral valve & $245(33)$ & $51(42.5)$ & $194(31.0)$ & 0.019 \\
\hline Aortic valve & $358(48)$ & $60(50.0)$ & $298(47.6)$ & 0.69 \\
\hline Tricuspid valve & $138(19)$ & $31(25.8)$ & $107(17.1)$ & 0.029 \\
\hline Pulmonary valve & $9(1.2)$ & 0 & $9(1.4)$ & 0.37 \\
\hline Surgery time, minutes & $342[277-428]$ & $351[300-443]$ & $340[273-426]$ & 0.25 \\
\hline Cardiopulmonary bypass & $650(87)$ & $106(88)$ & $544(87)$ & 0.77 \\
\hline Cardiopulmonary bypass time, minutes ${ }^{\mathrm{a}}$ & $178[135-231]$ & 199 [147-238] & 174 [134-229] & 0.040 \\
\hline Intraoperative fluid balance, $\mathrm{mL}$ & 2297 [1212-3335] & 2351 [1317-3208] & 2259 [1206-3347] & 0.83 \\
\hline APACHE II score & $13[11-15]$ & $13[11-15]$ & $13[11-15]$ & 0.098 \\
\hline \multicolumn{5}{|c|}{ Vasopressors and inotropes within the first $24 \mathrm{~h}$ after ICU admission } \\
\hline Norepinephrine, $n(\%)$ & $295(40)$ & $45(38)$ & $250(40)$ & 0.68 \\
\hline Maximal dose ${ }^{b}, \mu \mathrm{g} / \mathrm{kg} / \mathrm{min}$ & $0.08[0.05-0.14]$ & $0.06[0.04-0.12]$ & $0.08[0.05-0.14]$ & 0.51 \\
\hline Epinephrine, $n(\%)$ & $6(0.8)$ & $0(0)$ & $6(1.0)$ & 0.60 \\
\hline Maximal dose ${ }^{b}, \mu g / k g / m i n$ & $0.05[0.03-0.07]$ & 0 & $0.05[0.03-0.07]$ & \\
\hline Dopamine, $n(\%)$ & $128(17)$ & $16(13)$ & $112(18)$ & 0.29 \\
\hline Maximal dose ${ }^{b}, \mu g / k g / m i n$ & $3.1[1.9-4.1]$ & $3.6[1.9-4.8]$ & $3.0[1.9-4.1]$ & 0.52 \\
\hline Vasopressin, $n(\%)$ & $31(4.2)$ & $2(1.7)$ & $29(4.6)$ & 0.21 \\
\hline Phenylephrine, $n(\%)$ & $10(1.3)$ & 0 & $10(1.6)$ & 0.38 \\
\hline Dobutamine, $n(\%)$ & $406(53)$ & $74(62)$ & $332(53)$ & 0.089 \\
\hline Maximal dose ${ }^{b}, \mu g / k g / m i n$ & $2.6[1.7-3.5]$ & $3.0[1.6-3.7]$ & $2.6[1.7-3.5]$ & 0.31 \\
\hline PDE inhibitors, $n(\%)$ & $135(18)$ & $31(26)$ & $104(17)$ & 0.020 \\
\hline \multicolumn{5}{|c|}{ Blood products within the first $24 \mathrm{~h}$ after ICU admission, $n(\%)$} \\
\hline Red blood cells, units & $173(23)$ & $23(19)$ & $150(24)$ & 0.29 \\
\hline
\end{tabular}

IQR interquartile range, APACHE Acute Physiology and Chronic Health Evaluation, SOFA Sequential Organ Failure Assessment, AIDS acquired immunodeficiency syndrome, LVEF left ventricular ejection fraction, ICU intensive care unit, CABG coronary artery bypass grafting, PDE phosphodiesterase

${ }^{a}$ The denominator was patients in whom cardiopulmonary bypass surgery was conducted

${ }^{\mathrm{b}}$ The denominator was patients in whom each vasoactive agent was given during the first $24 \mathrm{~h}$ after ICU admission 
Table 2 Hemodynamic parameters and exposure variables

\begin{tabular}{|c|c|c|c|c|}
\hline & $\begin{array}{l}\text { All } \\
N=746\end{array}$ & $\begin{array}{l}\text { AKI group } \\
N=120\end{array}$ & $\begin{array}{l}\text { Non-AKI group } \\
N=626\end{array}$ & $p$ value \\
\hline Achieved MPP (time-weighted average), $\mathrm{mmHg}$ & $65[60-71]$ & $63[59-69]$ & $66[60-71]$ & 0.0047 \\
\hline Achieved MAP (time-weighted average), $\mathrm{mmHg}$ & 74 [69-79] & $72[68-78]$ & $74[69-79]$ & 0.051 \\
\hline Achieved CVP (time-weighted average), $\mathrm{mmHg}$ & $8.4[6.7-11]$ & $9.4[7.2-11]$ & $8.3[6.6-10]$ & 0.0048 \\
\hline Time spent with MPP $<60 \mathrm{mmHg}$, h & $6[2-13]$ & $7[4-13]$ & $5[2-12]$ & 0.0016 \\
\hline MPP-deficit (time-weighted average), \% & 20 [10-27] & $22[11-28]$ & 19 [10-27] & 0.41 \\
\hline Time spent with MPP-deficit $>20 \%, \mathrm{~h}$ & 12 [3-20] & $13[4-20]$ & $11[3-20]$ & 0.38 \\
\hline Time spent with MAP $<65 \mathrm{mmHg}$, h & $2[0-6]$ & $3[1-7]$ & $2[0-6]$ & 0.0090 \\
\hline MAP-deficit (time-weighted average), \% & $14[6.2-21]$ & $14[7.1-21]$ & $14[6.0-21]$ & 0.54 \\
\hline Time spent with MAP-deficit $>20 \%, \mathrm{~h}$ & $5[1-14]$ & $5[1-15]$ & $5[1-14]$ & 0.52 \\
\hline
\end{tabular}

$M A P$ mean arterial pressure, $C V P$ central venous pressure, $M P P$ mean perfusion pressure

MPP, while smaller relative hypotension was caused by lower preoperative or higher postoperative MPP. Since previous studies $[16,17,38]$ and our study have consistently shown that lower postoperative MPP is a risk factor of AKI, the non-linear relationship between preoperative MPP and AKI progression could weaken the association between relative hypotension and AKI progression. Higher preoperative MPP could increase the risk of AKI due to the right shift in the lower pressure limit of renal autoregulation in the presence of chronic hypertension [14]. On the contrary, lower preoperative MPP may also be a risk factor for postoperative AKI, especially in cardiac surgery patients. For example, chronic heart failure with reduced ejection fraction could result in low baseline MAP and is a risk factor for CSA-AKI [32, 39]. Patients with pulmonary hypertension and right heart failure could have high preoperative CVP and are at an increased risk of developing AKI [40]. Therefore, the effect of lower preoperative MPP on AKI among cardiac surgery patients may contribute to the different relationships of relative hypotension with AKI progression between cardiac surgery patients and other patient groups [21]. We report a lower preoperative MPP in our cohort $(80 \mathrm{mmHg})$ than the previous study enrolling cardiac surgery patients $(89 \mathrm{mmHg})$ [25], which may imply that we enrolled more patients with low preoperative MPP, resulting in a weaker correlation between relative hypotension and AKI progression. Moreover, we conducted multivariable logistic regression analyses, ensuring the robustness of our findings.

During the acute phase after cardiac surgery, routine CVP monitoring has helped guide hemodynamic management, such as detecting acute pericardial tamponade or right heart failure [41]. In addition to the utility in clinical practice, recent studies reported that elevated CVP is a prognostic indicator of poor outcomes in ICU patients. A meta-analysis reported the association of higher CVP with mortality and AKI among critically ill patients [13]. Observational studies showed that elevated CVP was a risk factor for mortality or AKI in cardiac surgery patients [42-44]. In line with these reports, we showed that increased CVP was associated with AKI progression. We also found that lower MPP was associated with AKI progression. Thus, out of the two components of MPP, i.e., MAP and CVP, CVP would substantially influence AKI progression in patients after cardiac surgery. As for the septic patients, CVP monitoring is not routine because CVP has been suggested as a poor indicator of fluid status. The previous large RCT for septic patients could not evaluate MPP [11]. However, a small observational study suggested that MPP might be associated with AKI [20]. Thus, in future studies for septic patients, reevaluation of CVP monitoring might be of value for preventing AKI progression.

\section{Significance and implications}

We report that although relative hypotension was not associated with AKI progression among patients after elective cardiac surgery, higher CVP and lower MPP were associated. Based on these results, we do not support adjusting MPP targets to the premorbid BP to prevent AKI progression after cardiac surgery. Since sequential CVP measurement is generally available using pulmonary artery catheters or central venous catheters after 

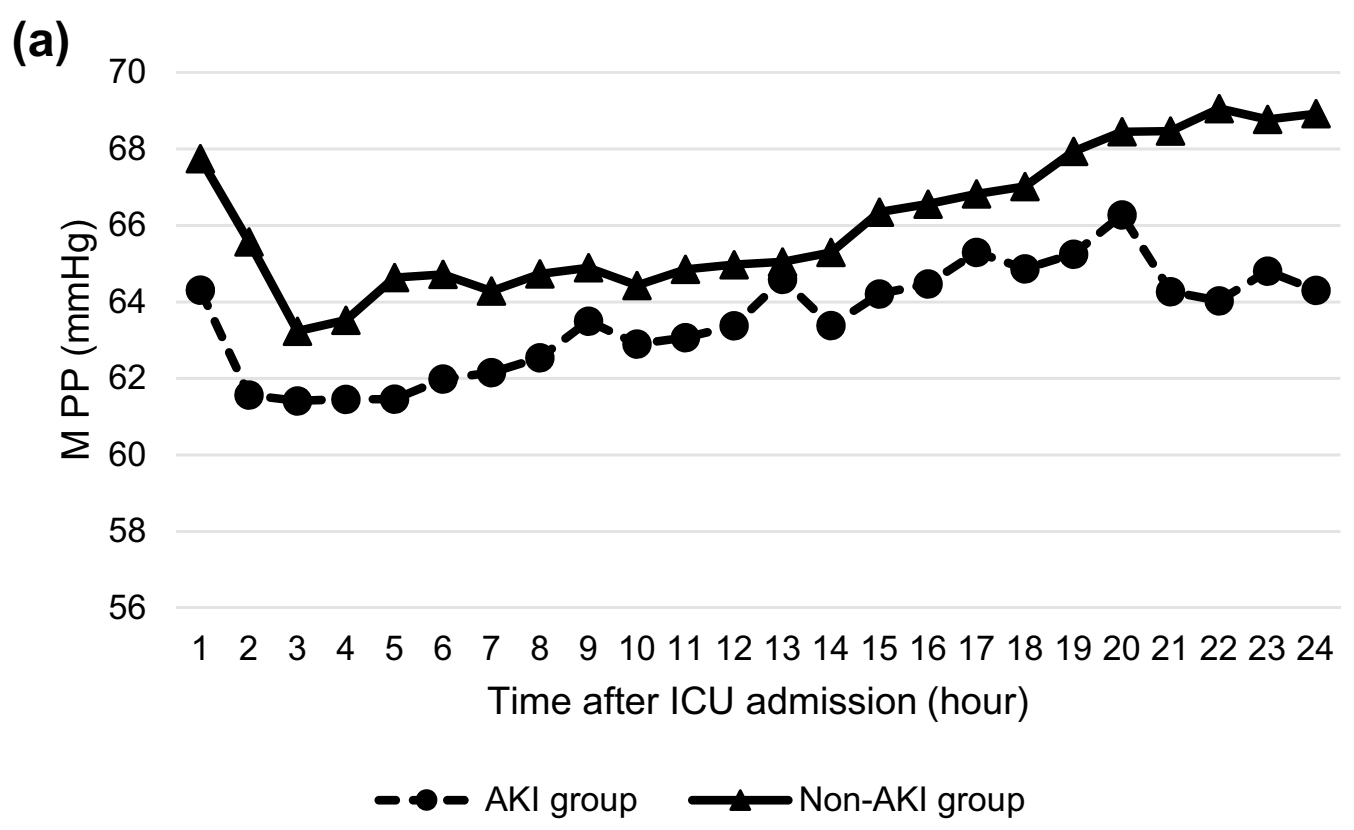

(b)

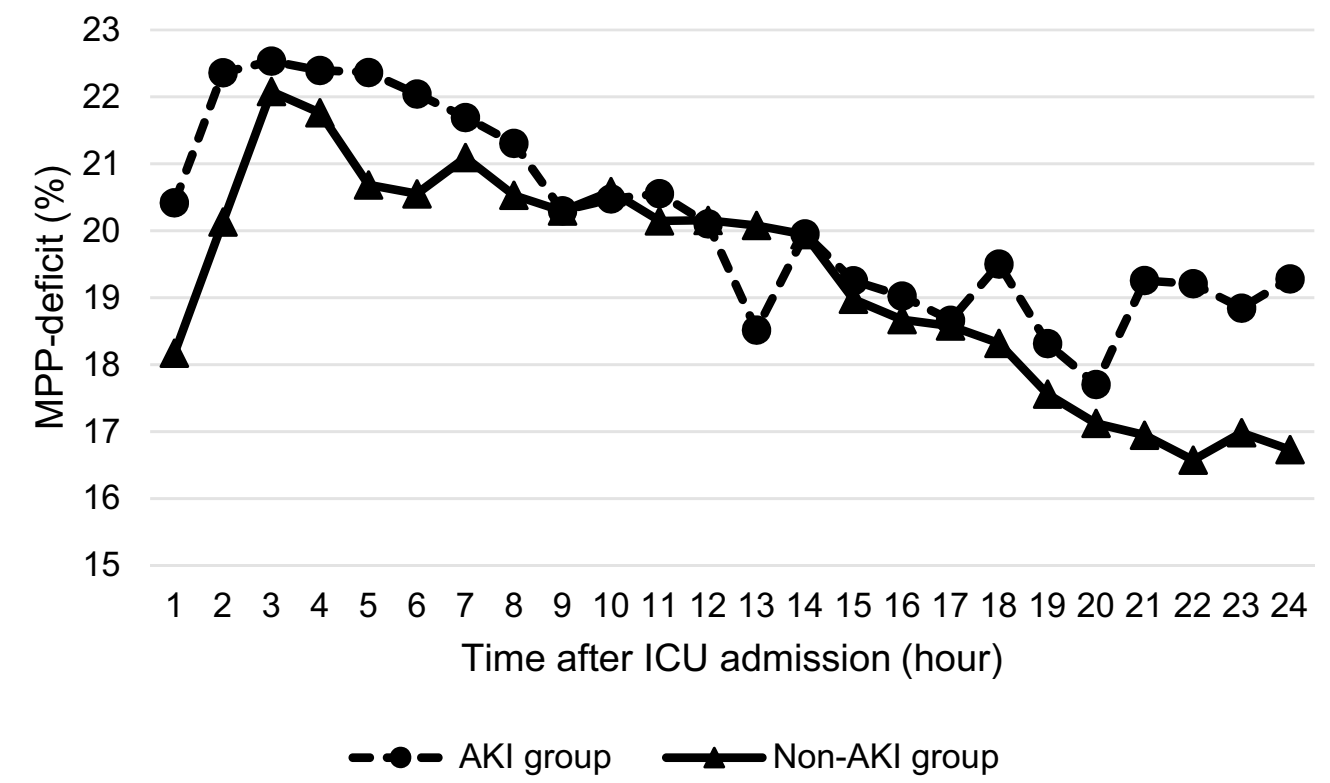

Fig. 1 Achieved mean perfusion pressure (a) and mean perfusion pressure-deficit (b) during the first $24 \mathrm{~h}$ after intensive care unit admission. AKI acute kidney injury, ICU intensive care unit, MPP mean perfusion pressure 
Table 3 Clinical outcomes

\begin{tabular}{lc}
\hline & All \\
& $\mathbf{N = 7 4 6}$ \\
\hline AKI progression between 24 and $72 \mathrm{~h}$ after ICU admission, $n(\%)$ & $120(16.1)$ \\
MAKE30, $n$ (\%) & $7(0.9)$ \\
Hospital mortality, $n$ (\%) & $4(0.5)$ \\
Need for renal replacement therapy during ICU stay, $n(\%)$ & $5(0.7)$ \\
Fluid balance on the second day of ICU stay, $\mathrm{mL}$ & $530.5[-86.3-1271.8]$ \\
Fluid balance on the third day of ICU stay ${ }^{\mathrm{a}}, \mathrm{mL}$ & $-429.0[-1240.0-100.0]$ \\
New-onset atrial fibrillation during ICU stay ${ }^{\mathrm{b}}, n(\%)$ & $86(14.9)$ \\
Stroke during hospital stay, $n$ (\%) & $12(1.6)$ \\
Mesenteric ischemia during hospital stay, $n(\%)$ & 0
\end{tabular}

$A K I$ acute kidney injury, MAKE major adverse kidney event, ICU intensive care unit, IQR interquartile range

${ }^{a}$ The denominator was patients who stayed in the ICU over 3 calendar days or more $(N=734)$

${ }^{b}$ The denominator was patients without a history of atrial fibrillation before ICU admission $(N=577)$

cardiac surgery, clinicians should pay more attention to high CVP values to identify patients at risk of AKI progression. In patients with elevated CVP, clinicians may consider avoiding interventions that can further increase the CVP value, such as excessive fluid resuscitation. Future studies are necessary to investigate whether lowering CVP values, such as with early diuretics administration, would decrease AKI progression among patients after cardiac surgery.

\section{Strengths and limitations}

This study is the first multicenter study assessing the relationship between relative hypotension and AKI progression in cardiac surgery patients using multivariable analyses adjusted for clinically relevant confounders. We had the largest sample size among the studies assessing relative hypotension and AKI in critically ill patients. In addition, there were no missing data in postoperative MAP at 1-h intervals, ensuring detailed and accurate BP data collection.

Our study has several limitations. First, time-weightedaverage BP may underestimate profound hypotension within a short time. However, we used the time spent with BP under a certain threshold as exposure variables to overcome the shortcoming of time-weighted-average BP. In addition, the associations of the two measures of exposure variable with AKI progression were consistent in all exposure variables, showing the robustness of the results. Second, we included some intraoperative confounders into the multivariable analyses on postoperative AKI progression. However, we did not collect BP data during the cardiac surgery. In addition, patients with AKI progression had longer cardiopulmonary bypass time. We may be missing some of the effects of intraoperative management. Future studies should deal with intraoperative variables in more detail. Third, as an observational cohort study, we could not control unmeasured or unknown confounders that may have influenced the results.

\section{Conclusions}

We found that relative hypotension is not associated with AKI progression, while higher CVP and lower MPP are associated with AKI progression in patients after cardiac surgery. However, these findings were based on exploratory investigation, and further studies for validating them are required. 
(a)
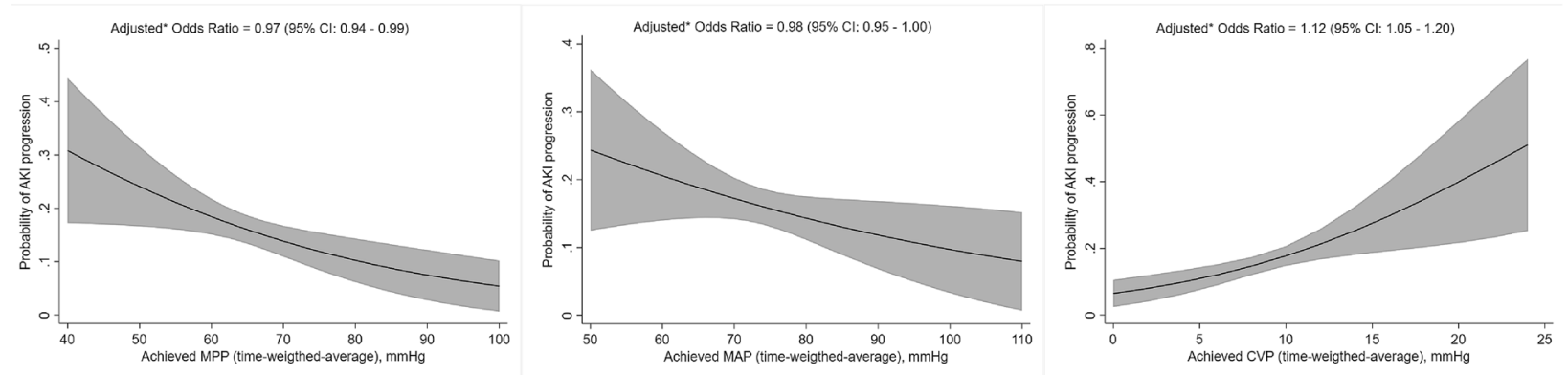

(b)
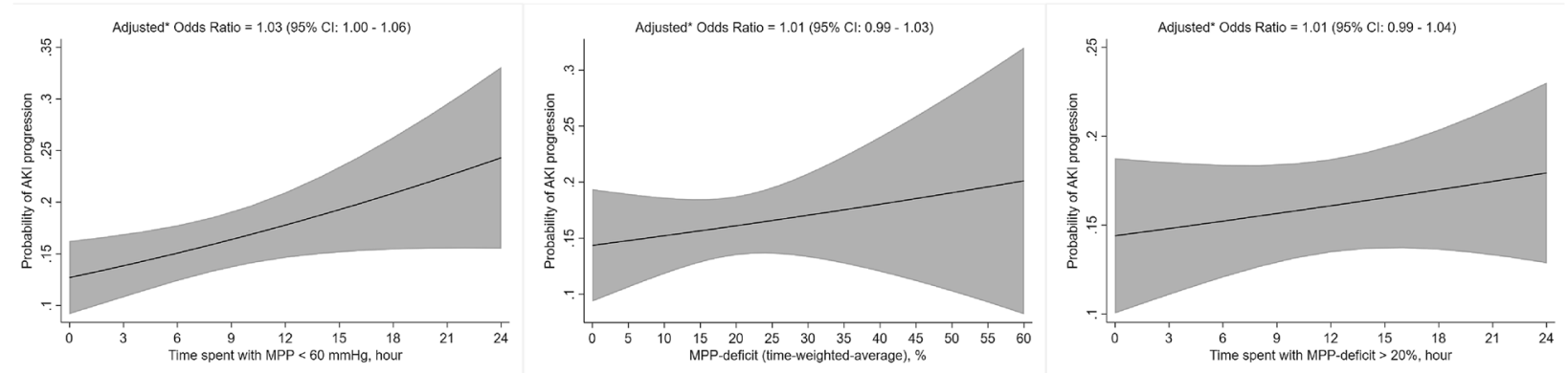

(c)
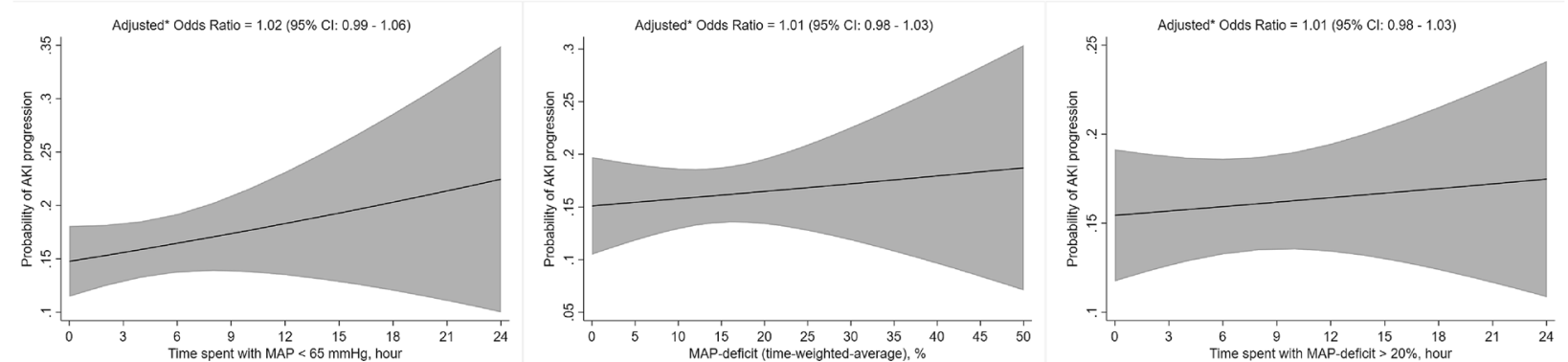

Fig. 2 Predicted marginal probabilities with 95\% confidence intervals for acute kidney injury progression versus exposure variables. a Achieved blood pressure. b Mean perfusion pressure. c Mean arterial pressure. AKl acute kidney injury, MAP mean arterial pressure, CVP central venous pressure, MPP mean perfusion pressure, Cl confidence interval

\section{Abbreviations}

AKI: Acute kidney injury; APACHE: Acute Physiology and Chronic Health Evaluation; BROTHER: Blood pressure and Relative Optimal Target after Heart surgery in Epidemiologic Registry; CABG: Coronary artery bypass grafting; Cl: Confidence interval; CPB: Cardiopulmonary bypass; CVP: Central venous pressure; ECMO: Extracorporeal membrane oxygenation; ICU: Intensive care unit; KDIGO: Kidney Disease: Improving Global Outcomes; MAP: Mean arterial pressure; MAKE: Major adverse kidney event; MPP: Mean perfusion pressure; OR: Odds ratio; RRT: Renal replacement therapy. 


\section{Supplementary Information}

The online version contains supplementary material available at https://doi. org/10.1186/s13613-021-00969-4.

Additional file 1: Table S1. Data collection and definitions of variables and outcomes. Table S2. The protocol to estimate preoperative central venous pressure. Table $\mathbf{S 3}$. Additional information on baseline characteristics and perioperative management. Table $\mathbf{S 4}$. Multivariable logistic regression analyses for acute kidney injury progression between 24 and $72 \mathrm{~h}$ after intensive care unit admission. Table S5. Sensitivity analyses for acute kidney injury progression between 24 and $72 \mathrm{~h}$ after intensive care unit admission. Figure $\mathbf{S 1}$. Details of data collection. Figure S2. Patient flow diagram. Figure S3. Achieved mean arterial pressure (a) and mean arterial pressure-deficit (b) during the first $24 \mathrm{~h}$ after intensive care unit admission

\section{Acknowledgements}

We would like to thank all the colleagues in the BROTHER study participating hospitals who performed the extensive data entry.

\section{The BROTHER study group}

Kameda Medical Center: Yuki Kotani; Jikei University School of Medicine: Takahiro Koga; Okinawa Chubu Hospital: Izumi Nakayama; Tottori University Faculty of Medicine: Masato Nakasone; Wakayama Medical University: Mami Shibata; Hokkaido University Hospital: Tomonao Yoshida; Kobe City Medical Center General Hospital: Akihisa Taguchi, Daisuke Kawakami; Hirosaki University Graduate School of Medicine: Noriko Mikami, Junichi Saito, Mirei Kubota, Tsubasa Oyama; Kurashiki Central Hospital: Jun Kamei; Yokohama City Minato Red Cross Hospital: Isao Nagata, Shinsuke Karatsu; Tokyo Bay Urayasu Ichikawa Medical Center: Naoki Yamaguchi; Yokohama City University Hospital: Takayuki Kariya; Iwate Prefectural Central Hospital: Hiroshi Nashiki; and Hiroshima Citizens Hospital, Hiroshima: Koji Kido, Hiroki Omiya.

\section{Authors' contributions}

YK had full access to all the data in the study and takes responsibility for the integrity of the data. Study concept and design: YK, TaY, JKu, KN, NT, and Jl. Acquisition of data: YK, TaY, JKa, AT, KK, NY, TKa, MN, NM, TKo, IN, MS, ToY, HN, and SK. Analysis and interpretation of data: YK, TaY, JKu, KN, NT, and Jl. Drafting of the manuscript: YK. Critical revision of the manuscript for important intellectual content: YK, TaY JKu, JKa, AT, KK, NY, TKa, MN, NM, TKo, IN, MS, ToY, HN, $S K, K N, N T$, and Jl. All authors read and approved the final manuscript.

\section{Funding}

None.

\section{Availability of data and materials}

The data that support the findings of this study are available from the corresponding author, TaY, upon reasonable request.

\section{Declarations}

\section{Ethics approval and consent to participate}

The ethics committee of Kameda Medical Center approved this study (No. 19-013), and all the ethics committees of participating hospitals approved an opt-out method of informed consent. Since this is a retrospective cohort study, we applied the opt-out method of informed consent for participation.

\section{Consent for publication}

Since this is a retrospective cohort study, we applied the opt-out method of informed consent for publication.

\section{Competing interests}

All the authors declare that they have no competing interests.

\section{Author details}

'Department of Intensive Care Medicine, Kameda Medical Center, Kamogawa, Japan. ${ }^{2}$ Department of Intensive Care Medicine, Tokyo Women's Medical University, 8-1 Kawada-cho, Shinjuku-ku, Tokyo 162-8666, Japan. ${ }^{3}$ Department of Critical Care Medicine, Sakai City Medical Center, Sakai, Japan. ${ }^{4}$ Emergency and Critical Care Center, Kurashiki Central Hospital, Kurashiki, Japan. ${ }^{5}$ Department of Anesthesiology and Critical Care, Kobe City Medical Center General Hospital, Kobe, Japan. ${ }^{6}$ Department of Anesthesiology and Intensive Care Medicine, Hiroshima Citizens Hospital, Hiroshima, Japan. ${ }^{7}$ Department of Emergency and Critical Care Medicine, Tokyo Bay Urayasu Ichikawa Medical Center, Urayasu, Japan. ${ }^{8}$ Intensive Care Department, Yokohama City University Medical Center, Yokohama, Japan. ${ }^{9}$ Division of Anesthesiology and Critical Care Medicine, Department of Surgery, Faculty of Medicine, Tottori University, Yonago, Japan. ${ }^{10}$ Department of Anesthesiology, Hirosaki University Graduate School of Medicine, Hirosaki, Japan. ${ }^{11}$ Intensive Care Unit, Department of Anesthesiology, Jikei University School of Medicine, Tokyo, Japan. ${ }^{12}$ Intensive Care Unit, Department of Internal Medicine, Okinawa Chubu Hospital, Okinawa, Japan. ${ }^{13}$ Department of Emergency and Critical Care Medicine, Wakayama Medical University, Wakayama, Japan. ${ }^{14}$ Department of Emergency Medicine, Hokkaido University Hospital, Sapporo, Japan. ${ }^{15}$ Intensive Care Unit, Iwate Prefectural Central Hospital, Morioka, Japan. ${ }^{16}$ Department of Intensive Care Medicine, Yokohama City Minato Red Cross Hospital, Yokohama, Japan. ${ }^{17}$ Cardiovascular Medicine, Nara Medical University, Kashihara, Japan. ${ }^{18}$ Department of Anesthesiology and Intensive Care, Osaka University Graduate School of Medicine, Suita, Japan. ${ }^{19}$ Department of Internal Medicine, Okinawa Prefectural Yaeyama Hospital Okinawa, Ishigaki, Japan.

Received: 26 August 2021 Accepted: 10 December 2021

Published online: 20 December 2021

\section{References}

1. Shimizu H, Okada M, Toh Y, et al. Thoracic and cardiovascular surgeries in Japan during 2018: annual report by the Japanese Association for Thoracic Surgery. Gen Thorac Cardiovasc Surg. 2021;69:179-212.

2. Bowdish ME, D'Agostino RS, Thourani VH, et al. STS adult cardiac surgery database: 2021 update on outcomes, quality, and research. Ann Thorac Surg. 2021;111(6):1770-80.

3. Gurfinkel EP, Lernoud VS, Laguens RP, et al. Advances in coronary heart disease surgery in Latin America. Circulation. 2007;115:1147-53.

4. Head SJ, Howell NJ, Osnabrugge RL, et al. The European association for cardio-thoracic surgery (EACTS) database: an introduction. Eur J Cardiothorac Surg. 2013;44:e175-80.

5. Rao C, Zhang H, Gao H, et al. The Chinese cardiac surgery registry: design and data audit. Ann Thorac Surg. 2016;101:1514-20.

6. Mao H, Katz N, Ariyanon W, et al. Cardiac surgery-associated acute kidney injury. Cardiorenal Med. 2013;3:178-99.

7. Hobson CE, Yavas S, Segal MS, et al. Acute kidney injury is associated with increased long-term mortality after cardiothoracic surgery. Circulation. 2009;119:2444-53.

8. Ortega-Loubon C, Fernández-Molina M, Carrascal-Hinojal Y, et al. Cardiac surgery-associated acute kidney injury. Ann Card Anaesth. 2016:19:687-98.

9. Brown JR, Kramer RS, Coca SG, et al. Duration of acute kidney injury impacts long-term survival after cardiac surgery. Ann Thorac Surg. 2010:90:1142-8.

10. Wang Y, Bellomo R. Cardiac surgery-associated acute kidney injury: risk factors, pathophysiology and treatment. Nat Rev Nephrol. 2017;13:697-711.

11. Asfar P, Meziani F, Hamel JF, et al. High versus low blood-pressure target in patients with septic shock. N Engl J Med. 2014;370:1583-93.

12. Lamontagne F, Richards-Belle A, Thomas K, et al. Effect of reduced exposure to vasopressors on 90-day mortality in older critically ill patients with vasodilatory hypotension: a randomized clinical trial. JAMA. 2020;323:938-49.

13. Chen $C Y$, Zhou $Y$, Wang $P$, et al. Elevated central venous pressure is associated with increased mortality and acute kidney injury in critically ill patients: a meta-analysis. Crit Care. 2020;24:80.

14. Panwar R. Untreated relative hypotension measured as perfusion pressure deficit during management of shock and new-onset acute kidney injury - a literature review. Shock. 2018;49:497-507.

15. Gambardella I, Gaudino M, Ronco C, et al. Congestive kidney failure in cardiac surgery: the relationship between central venous pressure and acute kidney injury. Interact Cardiovasc Thorac Surg. 2016;23:800-5. 
16. Gül I, Cerit L, Senturk B, et al. The negative effect of mean perfusion pressure on the development of acute kidney injury after transcatheter aortic valve implantation. Braz J Cardiovasc Surg. 2018;33:559-66.

17. Neuman J, Schulz L, Aneman A. Associations between mean systemic filling pressure and acute kidney injury: an observational cohort study following cardiac surgery. Acta Anaesthesiol Scand. 2021;65:373-80.

18. KDIGO. KDIGO clinical practice guideline for acute kidney injury. Kidney Int Suppl. 2012;2:1-138.

19. Panwar R, Lanyon N, Davies AR, et al. Mean perfusion pressure deficit during the initial management of shock-an observational cohort study. J Crit Care. 2013:28:816-24.

20. Wong BT, Chan MJ, Glassford NJ, et al. Mean arterial pressure and mean perfusion pressure deficit in septic acute kidney injury. J Crit Care. 2015;30:975-81.

21. Panwar R, Tarvade S, Lanyon N, et al. Relative hypotension and adverse kidney-related outcomes among critically ill patients with shock. A multicenter, prospective cohort study. Am J Respir Crit Care Med. 2020;202:1407-18.

22. Gillinov AM, Bagiella E, Moskowitz AJ, et al. Rate control versus rhythm control for atrial fibrillation after cardiac surgery. N Engl J Med. 2016;374:1911-21.

23. Landoni G, Lomivorotov WV, Nigro Neto C, et al. Volatile anesthetics versus total intravenous anesthesia for cardiac surgery. N Engl J Med. 2019;380:1214-25.

24. Mazer CD, Whitlock RP, Fergusson DA, et al. Restrictive or liberal red-cell transfusion for cardiac surgery. N Engl J Med. 2017;377:2133-44.

25. Saito S, Uchino S, Takinami M, et al. Postoperative blood pressure deficit and acute kidney injury progression in vasopressor-dependent cardiovascular surgery patients. Crit Care. 2016;20:74.

26. Knaus WA, Draper EA, Wagner DP, et al. APACHE II: a severity of disease classification system. Crit Care Med. 1985;13:818-29.

27. Vincent JL, Moreno R, Takala J, et al. The SOFA (sepsis-related organ failure assessment) score to describe organ dysfunction/failure. On behalf of the working group on sepsis-related problems of the European society of intensive care medicine. Intensive Care Med. 1996;22:707-10.

28. Beigel R, Cercek B, Luo H, et al. Noninvasive evaluation of right atrial pressure. J Am Soc Echocardiogr. 2013;26:1033-42.

29. Damman K, van Deursen VM, Navis G, et al. Increased central venous pressure is associated with impaired renal function and mortality in a broad spectrum of patients with cardiovascular disease. J Am Coll Cardiol. 2009;53:582-8.

30. Semler MW, Rice TW, Shaw AD, et al. Identification of major adverse kidney events within the electronic health record. J Med Syst. 2016;40:167.

31. Hoste EA, Bagshaw SM, Bellomo R, et al. Epidemiology of acute kidney injury in critically ill patients: the multinational AKI-EPI study. Intensive Care Med. 2015;41:1411-23.

32. Coleman MD, Shaefi S, Sladen RN. Preventing acute kidney injury after cardiac surgery. Curr Opin Anaesthesiol. 2011;24:70-6.

33. Cheungpasitporn W, Thongprayoon C, Kittanamongkolchai W, et al. Comparison of renal outcomes in off-pump versus on-pump coronary artery bypass grafting: a systematic review and meta-analysis of randomized controlled trials. Nephrology. 2015;20:727-35.

34. Khan UA, Coca SG, Hong K, et al. Blood transfusions are associated with urinary biomarkers of kidney injury in cardiac surgery. J Thorac Cardiovasc Surg. 2014;148:726-32.

35. Chertow GM, Lazarus JM, Christiansen CL, et al. Preoperative renal risk stratification. Circulation. 1997;95:878-84.

36. Wahba A, Milojevic M, Boer C, et al. 2019 EACTS/EACTA/EBCP guidelines on cardiopulmonary bypass in adult cardiac surgery. Eur J Cardiothorac Surg. 2019;57:210-51.

37. Strandgaard S, Olesen J, Skinhoj E, et al. Autoregulation of brain circulation in severe arterial hypertension. Br Med J. 1973;1:507-10.

38. Ostermann M, Hall A, Crichton S. Low mean perfusion pressure is a risk factor for progression of acute kidney injury in critically ill patients-a retrospective analysis. BMC Nephrol. 2017;18:151.

39. Cautela J, Tartiere J-M, Cohen-Solal A, et al. Management of low blood pressure in ambulatory heart failure with reduced ejection fraction patients. Eur J Heart Fail. 2020;22:1357-65.

40. Bansal S, Prasad A, Linas S. Right heart failure-unrecognized cause of cardiorenal syndrome. J Am Soc Nephrol. 2018;29:1795-8.
41. Shabetai R. Pericardial effusion: haemodynamic spectrum. Heart. 2004;90:255-6.

42. Williams JB, Peterson ED, Wojdyla D, et al. Central venous pressure after coronary artery bypass surgery: does it predict postoperative mortality or renal failure? J Crit Care. 2014;29:1006-10.

43. Lopez MG, Shotwell MS, Morse J, et al. Intraoperative venous congestion and acute kidney injury in cardiac surgery: an observational cohort study. Br J Anaesth. 2021;126:599-607.

44. McCoy IE, Montez-Rath ME, Chertow GM, et al. Central venous pressure and the risk of diuretic-associated acute kidney injury in patients after cardiac surgery. Am Heart J. 2020;221:67-73.

\section{Publisher's Note}

Springer Nature remains neutral with regard to jurisdictional claims in published maps and institutional affiliations.

\section{Submit your manuscript to a SpringerOpen ${ }^{\circ}$ journal and benefit from:}

- Convenient online submission

- Rigorous peer review

- Open access: articles freely available online

- High visibility within the field

- Retaining the copyright to your article

Submit your next manuscript at $\boldsymbol{\nabla}$ springeropen.com 